\title{
Performance Analysis of Hybrid Cognitive Radio Systems with Imperfect Channel Knowledge
}

\author{
Ankit Kaushik $^{*}$, Shree Krishna Sharma ${ }^{\dagger}$, Symeon Chatzinotas $^{\dagger}$, Björn Ottersten ${ }^{\dagger}$, Friedrich Jondral ${ }^{*}$ \\ ${ }^{*}$ Communications Engineering Lab, Karlsruhe Institute of Technology (KIT), Germany \\ \{ankit.kaushik, friedrich.jondral\}@kit.edu \\ ${ }^{\dagger}$ SnT - securityandtrust.lu, University of Luxembourg, Luxembourg \\ \{shree.sharma, symeon.chatzinotas, bjorn.ottersten\}@uni.lu
}

\begin{abstract}
In this paper, we study the performance of hybrid cognitive radio systems that combine the benefits of interweave and underlay systems by employing a spectrum sensing and a power control mechanism at the Secondary Transmitter (ST). Existing baseline models considered for performance analysis assume perfect knowledge of the involved channels at the ST, however, such situations hardly exist in practical deployments. Motivated by this fact, we propose a novel approach that incorporates channel estimation at the ST, and consequently characterizes the performance of Hybrid Systems (HSs) under realistic scenarios. To capture the impact of imperfect channel knowledge, we propose outage constraints on the detection probability at the ST and on the interference power received at the primary receiver. Our analysis reveals that the baseline model overestimates the performance of the HS in terms of achievable secondary user throughput. Finally, based on the proposed estimation-sensing-throughput tradeoff, we determine suitable estimation and sensing durations that effectively capture the effect of imperfect channel knowledge and subsequently enhance the achievable secondary user throughput.
\end{abstract}

\section{INTRODUCTION}

Cognitive Radio (CR) communication is considered as one of the potential solutions that addresses the spectrum scarcity problem of future wireless networks. According to Goldsmith et al. [1], secondary access to the licensed spectrum can be associated with different CR paradigms, which are employed by the secondary system. These paradigms include interweave, underlay and overlay systems. Since interweave and underlay systems are mainly associated with the physical layer, they are considered for hardware implementations. According to interweave systems, Secondary Users (SUs) utilize the licensed spectrum opportunistically by exploiting spectral holes in different domains such as time, frequency, space, polarization, etc, whereas in underlay systems, SUs are allowed to use the primary spectrum as long as they respect the interference constraints of the Primary Receivers (PRs).

Underlay systems employ several techniques such as power control, interference alignment, beamforming that allow CR systems to mitigate the interference at the primary systems [2]. More particularly, the underlay systems tend to operate below a certain level defined as the Interference Threshold (IT). In the context of an interweave system, the interference is avoided by sensing a licensed spectrum or Primary User (PU) signal

This work was partially supported by the National Research Fund, Luxembourg under the CORE projects "SeMIGod" and "SATSENT". at the Secondary Transmitter (ST). Spectrum sensing can be performed by employing various techniques such as energy detection, matched filter based detection, cyclostationary based detection [2]. Due to its versatility towards the primary signal, we consider energy detection for performing spectrum sensing. In this view, the performance of interweave systems depend on the detector's performance, which is characterized in terms of detection probability and false alarm probability. As a result, to ensure that the interference is restricted below a certain level, it is essential to operate the detector in such a way that the detection probability stays above a desired level. Besides that, the performance of the secondary system can be characterized in terms of throughput achieved at the Secondary Receiver (SR), which is generally influenced by the false alarm probability. In this context, a fundamental relationship between the sensing and the SU throughput has been investigated by Liang et al. [3].

However, the interweave system does not account for the severity of the interference power received at the PR, which in most cases can be tolerated by the primary systems and in other cases can lead to outage at the PR, thereby resulting in serious performance degradation of the primary system. In contrast to the interweave systems, the detection incapability of underlay systems forbids them to transmit with full power, specially during the periods when the primary system remains inactive. Addressing these issues can significantly enhance the spectral efficiency of the CR systems. In this context, a joint solution that utilizes the interference tolerance capability of the underlay systems and the agility of interweave systems to detect spectrum holes, defined as Hybrid System (HS), has been proposed [4]-[8].

Kang et al. [4] established a frame structure for HSs, whereby the ST first senses a PU channel in order to decide its operation mode (interweave or underlay) based on the detection result. Further, to decide upon a suitable operation mode, appropriate strategies that maximize the secondary system's throughput have been investigated by Oh et al. [5] and Senthuran et al. [6], respectively. Besides that, Jiang et al. employed a double detection threshold, which enables dynamic switching between full and partial access modes. Lastly, a sensing-throughput tradeoff to characterize the performance of the HS has been investigated by Sharma et al. [8]. To the best of our knowledge, most of the existing 


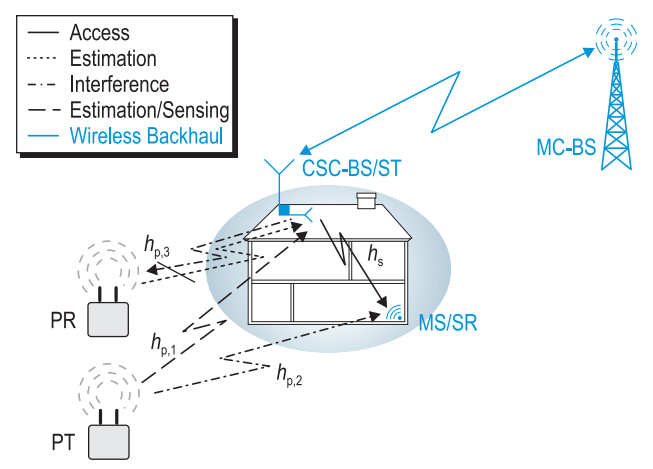

Fig. 1. A cognitive small cell scenario demonstrating: (i) the underlay paradigm, (ii) the associated network elements, which constitute Cognitive Small Cell-Base Station/Secondary Transmitter (CSC-BS/ST), Mobile Station/Secondary Receiver (MS/SR), Macro Cell-Base Station (MC-BS) and Primary Transmitter (PT), (iii) the interacting channels: sensing $\left(h_{\mathrm{p}, 1}\right)$, interference $\left(h_{\mathrm{p}, 2}, h_{\mathrm{p}, 3}\right)$ and access $\left(h_{\mathrm{s}}\right)$ channels.

models used for performance analysis assume the perfect knowledge of the involved channels at the ST. This assumption is however not viable for practical implementations, thereby rendering the performance analysis carried out using these models inaccurate. In this context, the performance analysis of the HS that incorporates channel estimation is an interesting research problem. Motivated by this fact, we establish a framework that considers the estimation of the involved channels and characterizes the impact of estimation errors on CR system performance. More specifically, this paper provides the following contributions:

- We establish a novel analytical framework for the HS that constitutes the estimation of interacting channels, namely: (i) sensing channel, (ii) interference channels and (iii) access channel.

- Based on the proposed framework, we investigate the impact of imperfect channel knowledge in terms of the interference encountered at the PR. Particularly, to restrict this interference, we employ outage constraints on the detection probability at the ST and on the interference power received at the PR. Consequently, we analyze the performance of the HS in terms of the achievable SU throughput.

- Finally, we depict a fundamental tradeoff between estimation time, sensing time and achievable throughput. We exploit this tradeoff to determine suitable estimation and sensing durations that achieve a maximum performance for the HS.

\section{System Model}

The Cognitive Small Cell (CSC), a CR application, characterizes a small cell deployment that fulfills the spectral requirements for Mobile Stations (MSs) operating indoor, cf. Fig. 1. For the disposition of the CSC in the network, the following key elements are essential: a CSC-Base Station (CSC-BS), a Macro Cell-Base Station (MC-BS) and MS [9]. Considering the fact that the spectrum sensing and power control are employed at the CSC-BS, the CSC-BS and the MS represent ST and SR, respectively.
As an extension to the existing models depicted in [4], [8], we consider a slotted medium access for the HS where the time axis is segmented into frames of length $T$, according to which, the ST employs periodic sensing. In this view, each frame consists of a sensing interval $\tau_{\text {sen }}$ followed by data transmission $\left(T-\tau_{\mathrm{sen}}\right)$. Depending on the outcome of the sensing, the data transmission takes place with or without power control.

\section{A. Signal model}

We consider that both primary and secondary systems employ Orthogonal Frequency Division Multiplex (OFDM) signal to carry out their transmissions. As a result, OFDM signals transmitted by the primary system are modeled as zero mean Gaussian signals by the secondary system, and viceversa. Subject to the underlying hypothesis, illustrating the presence $\mathcal{H}_{1}$ and the absence $\mathcal{H}_{0}$ of the primary signal, the discrete and complex received signal is given by

$$
y_{\mathrm{ST}}[n]=\left\{\begin{array}{ll}
h_{\mathrm{p}, 1} \cdot x_{\mathrm{PT}}[n]+w[n] & : \mathcal{H}_{1} \\
w[n] & : \mathcal{H}_{0}
\end{array},\right.
$$

where $x_{\mathrm{PT}}[n]$ corresponds to a discrete and complex sample transmitted by the PT, $\left|h_{\mathrm{p}, 1}\right|^{2}$ represents the power gain of the sensing channel for a given frame and $w[n]$ is circularly symmetric additive white Gaussian noise at the ST. The mean and variance for the signal and the noise are determined as: $\mathbb{E}\left[x_{\mathrm{PT}}[n]\right]=0, \mathbb{E}[w[n]]=0, \mathbb{E}\left[\left|x_{\mathrm{PT}}[n]\right|^{2}\right]=\sigma_{\mathrm{s}}^{2}$ and $\mathbb{E}\left[|w[n]|^{2}\right]=\sigma_{\mathrm{w}}^{2}$. The channel $h_{\mathrm{p}, 1}$ is considered to be independent of $x_{\mathrm{PT}}[n]$ and $w[n]$, thus, $y_{\mathrm{ST}}[n]$ is also an independent and identically distributed (i.i.d.) random process.

Following the conventional frame structure, ST performs sensing for a duration of $\tau_{\text {sen }}$. The test statistics $\mathrm{T}(\mathbf{y})$ at the ST is evaluated as $\mathbf{T}(\mathbf{y})=\frac{1}{\tau_{\text {sen } f_{\mathrm{s}}}} \sum_{n}^{\tau_{\text {sen }} f_{\mathrm{s}}}\left|y_{\mathrm{ST}}[n]\right|^{2} \gtrless_{\mathcal{H}_{0}}^{\mathcal{H}_{1}} \mu$, where $\mu$ is the decision threshold and $\mathbf{y}$ is a vector with $\tau_{\text {sen }} f_{\mathrm{s}}$ samples. $\mathrm{T}(\mathbf{y})$ represents a random variable, whereby the characterization of the distribution function depends on the underlying hypothesis. Corresponding to the considered PU signal model, $\mathrm{T}(\mathbf{y})$ follows a central chi-squared $\left(\mathcal{X}^{2}\right)$ distribution for both hypotheses $\mathcal{H}_{0}$ and $\mathcal{H}_{1}$ [10]. Consequently, the detection probability $\left(\mathrm{P}_{\mathrm{d}}\right)$ and the false alarm probability $\left(\mathrm{P}_{\mathrm{fa}}\right)$ are determined as [11]

$$
\begin{aligned}
\mathrm{P}_{\mathrm{d}}\left(\mu, \tau_{\mathrm{sen}}, P_{\mathrm{Rx}, \mathrm{ST}, h_{\mathrm{p}, 1}}\right) & =\Gamma\left(\frac{\tau_{\mathrm{sen}} f_{\mathrm{s}}}{2}, \frac{\tau_{\mathrm{sen}} f_{\mathrm{s}} \mu}{2 P_{\mathrm{Rx}, \mathrm{ST}, h_{\mathrm{p}, 1}}}\right), \\
\mathrm{P}_{\mathrm{fa}}\left(\mu, \tau_{\mathrm{sen}}\right) & =\Gamma\left(\frac{\tau_{\mathrm{sen}} f_{\mathrm{s}}}{2}, \frac{\tau_{\mathrm{sen}} f_{\mathrm{s}} \mu}{2 \sigma_{\mathrm{w}}^{2}}\right),
\end{aligned}
$$

where $P_{\mathrm{Rx}, \mathrm{ST}, h_{\mathrm{p}, 1}}$ is the power received over the sensing channel and $\Gamma(\cdot, \cdot)$ represents a regularized upper incomplete Gamma function [12].

Similar to (1), the discrete and complex received signal at the SR conditioned on the sensing outcome is given by

$y_{\mathrm{SR}}[n]= \begin{cases}h_{\mathrm{s}} \cdot x_{\mathrm{ST}, \text { full }}[n]+h_{\mathrm{p}, 2} \cdot x_{\mathrm{PT}}[n]+w[n] & : 1-\mathrm{P}_{\mathrm{d}} \\ h_{\mathrm{s}} \cdot x_{\mathrm{ST}, \text { full }}[n]+w[n] & : 1-\mathrm{P}_{\mathrm{fa}} \\ h_{\mathrm{s}} \cdot x_{\mathrm{ST}, \text { cont }}[n]+h_{\mathrm{p}, 2} \cdot x_{\mathrm{PT}}[n]+w[n] & : \mathrm{P}_{\mathrm{d}} \\ h_{\mathrm{s}} \cdot x_{\mathrm{ST}, \text { cont }}[n]+w[n] & : \mathrm{P}_{\mathrm{fa}}\end{cases}$ 
where $x_{\mathrm{ST}, \text { full }}[n]$ and $x_{\mathrm{ST}, \mathrm{cont}}[n]$ present the discrete and complex samples with full transmit power $P_{\mathrm{Tx}, \mathrm{ST} \text {,full }}$ and controlled transmit power $P_{\mathrm{Tx}, \mathrm{ST}, \mathrm{cont}}$, respectively. Additionally, $\left|h_{\mathrm{s}}\right|^{2}$ and $\left|h_{\mathrm{p}, 2}\right|^{2}$ represent the power gains for the access and the interference channels, cf. Fig. 1.

Besides, an interference from the ST is encountered at the PR across the channel $h_{\mathrm{p}, 3}$ only for the cases where the PT is transmitting, i.e., $\left(1-\mathrm{P}_{d}, \mathrm{P}_{d}\right)$, cf. (4). In this regard, the interference signal from the $\mathrm{ST}$ at the $\mathrm{PR}$ is given by

$$
y_{\mathrm{PR}}[n]=\left\{\begin{array}{ll}
h_{\mathrm{p}, 3} \cdot x_{\mathrm{ST}, \mathrm{cont}}[n]+w[n] & : \mathbf{P}_{\mathrm{d}} \\
h_{\mathrm{p}, 3} \cdot x_{\mathrm{ST}, \text { full }}[n]+w[n] & : 1-\mathbf{P}_{\mathrm{d}}
\end{array} .\right.
$$

\section{B. Problem Description}

To employ a power control mechanism, the ST is required to control its transmit power in such a way that the interference power received at the $\mathrm{PR}$ is below a certain interference threshold $\left(\theta_{\mathrm{I}}\right)$. In reference to the HS, constraints on interference power received at the PR are defined as

$$
\begin{array}{r}
\mathbb{P}\left(\mathcal{H}_{1}\right) \cdot \mathrm{P}_{\mathrm{d}} \cdot\left|h_{\mathrm{p}, 3}\right|^{2} P_{\mathrm{Tx}, \mathrm{ST}, \text { cont }} \leq \theta_{\mathrm{I}} \\
\text { and } \mathbb{P}\left(\mathcal{H}_{1}\right) \cdot\left(1-\mathrm{P}_{\mathrm{d}}\right) \cdot\left|h_{\mathrm{p}, 3}\right|^{2} P_{\mathrm{Tx}, \mathrm{ST}, \text { full }} \leq \theta_{\mathrm{I}},
\end{array}
$$

where $\mathbb{P}\left(\mathcal{H}_{1}\right)\left(=1-\mathbb{P}\left(\mathcal{H}_{0}\right)\right)$ represents the occurrence probability of the hypothesis $\mathcal{H}_{1}$. According to [8], (7) is usually handled by the regulatory bodies. In this regard, using (6) and the knowledge of $\theta_{\mathrm{I}}$, the controlled power is computed as $\frac{\theta_{\mathrm{I}}}{\mathbb{P}\left(\mathcal{H}_{1}\right) \cdot \mathrm{P}_{\mathrm{d}} \cdot\left|h_{\mathrm{p}, 3}\right|^{2}}$.

Next, the throughput received at the SR corresponding to the cases illustrated in (4) is characterized. Subject to the sensing outcome $1-\mathrm{P}_{\mathrm{fa}}, 1-\mathrm{P}_{\mathrm{d}}, \mathrm{P}_{\mathrm{fa}}, \mathrm{P}_{\mathrm{d}}$, the corresponding throughputs at the SR are defined as

$$
\begin{aligned}
& R_{0}\left(\tau_{\text {sen }}\right)=\frac{T-\tau_{\text {sen }}}{T} \cdot \overbrace{\log _{2}\left(1+\left|h_{\mathrm{s}}\right|^{2} \frac{P_{\mathrm{Tx}, \mathrm{ST}, \text { full }}}{\sigma_{\mathrm{w}}^{2}}\right)}^{\mathrm{C}_{0}} \\
& \times\left(1-\mathrm{P}_{\mathrm{fa}}\right) \cdot \mathbb{P}\left(\mathcal{H}_{0}\right), \\
& R_{1}\left(\tau_{\text {sen }}\right)=\frac{T-\tau_{\text {sen }}}{T} \overbrace{\log _{2}\left(1+\frac{\left|h_{\mathrm{s}}\right|^{2} P_{\mathrm{Tx}, \mathrm{ST}, \text { full }}}{\left|h_{\mathrm{p}, 2}\right|^{2} \sigma_{\mathrm{s}}^{2}+\sigma_{\mathrm{w}}^{2}}\right)}^{\mathrm{C}_{1}} \\
& \times\left(1-\mathrm{P}_{\mathrm{d}}\right) \cdot \mathbb{P}\left(\mathcal{H}_{1}\right), \\
& R_{2}\left(\tau_{\text {sen }}\right)=\frac{T-\tau_{\text {sen }}}{T} \cdot \overbrace{\log _{2}\left(1+\left|h_{\mathrm{s}}\right|^{2} \frac{P_{\mathrm{Tx}, \mathrm{ST}, \mathrm{cont}}}{\sigma_{\mathrm{w}}^{2}}\right)}^{\mathrm{C}_{2}} \\
& \times \mathrm{P}_{\mathrm{fa}} \cdot \mathbb{P}\left(\mathcal{H}_{0}\right), \\
& R_{3}\left(\tau_{\text {sen }}\right)=\frac{T-\tau_{\text {sen }}}{T} \cdot \overbrace{\log _{2}\left(1+\frac{\left|h_{\mathrm{s}}\right|^{2} P_{\mathrm{Tx}, \mathrm{ST}, \mathrm{cont}}}{\left|h_{\mathrm{p}, 2}\right|^{2} \sigma_{\mathrm{s}}^{2}+\sigma_{\mathrm{w}}^{2}}\right)}^{\mathrm{C}_{3}} \\
& \times \mathrm{P}_{\mathrm{d}} \cdot \mathbb{P}\left(\mathcal{H}_{1}\right) \text {. }
\end{aligned}
$$

where $\mathrm{C}_{0}, \mathrm{C}_{1}, \mathrm{C}_{2}$ and $\mathrm{C}_{3}$ represent the date rates.

Sharma et. al. [8] established a tradeoff between the sensing time and $\mathrm{SU}$ throughput $\left(R_{\mathrm{s}}\right)$ subject to a target detection

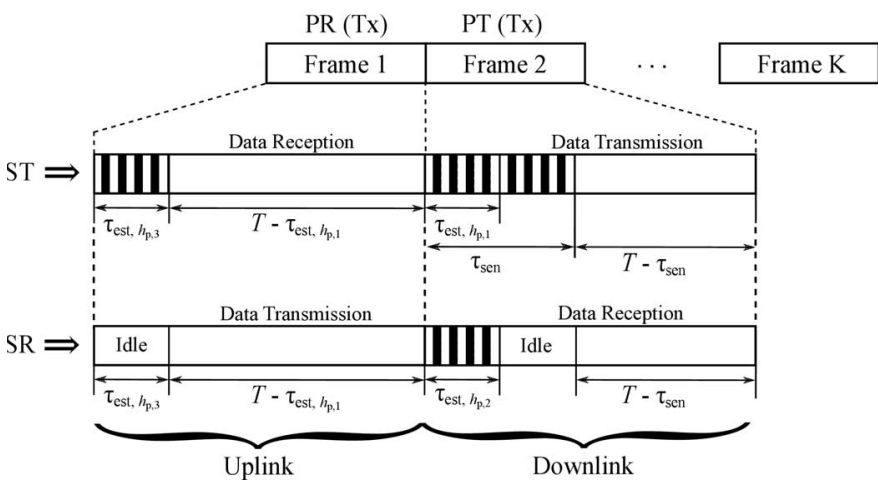

Fig. 2. Frame structure of the HSs illustrating the time allocation for channel estimation, sensing and data transmission from the perspective of a ST and a SR.

probability $\left(\overline{\mathrm{P}}_{\mathrm{d}}\right)$. This tradeoff is represented as

$$
\begin{aligned}
R_{\mathrm{S}}\left(\tilde{\tau}_{\text {sen }}\right) & =\max _{\tau_{\text {sen }}} R_{\mathrm{s}}\left(\tau_{\text {sen }}\right) \\
& =R_{0}\left(\tau_{\text {sen }}\right)+R_{1}\left(\tau_{\text {sen }}\right)+R_{2}\left(\tau_{\text {sen }}\right)+R_{3}\left(\tau_{\text {sen }}\right) \\
\text { s.t. } & \mathrm{P}_{\mathrm{d}} \geq \overline{\mathrm{P}}_{\mathrm{d}} .
\end{aligned}
$$

As a consequence, the tradeoff depicted in (12) determines a suitable sensing time $\tilde{\tau}_{\text {sen }}$ that achieves the maximum SU throughput. However, the system model depicted above has the following fundamental issues:

- Without the knowledge of received power (sensing channel, $\left.h_{\mathrm{p}, 1}\right)$, the characterization of $\mathrm{P}_{\mathrm{d}}$ is not possible, cf. (2). This leaves the constraint defined in (13) inappropriate.

- Without the knowledge of the interference channel towards the PR $\left(h_{\mathrm{p}, 3}\right)$, the power control mechanism cannot be employed at the ST.

- Along with the above mentioned channels, the knowledge of the access $\left(h_{\mathrm{s}}\right)$ and the interference channel $\left(h_{\mathrm{p}, 2}\right)$ to the $\mathrm{SR}$, from the PT, is required at the ST for characterizing the SU throughput.

With these issues, it is not reasonable to consider the performance analysis depicted by the ideal model (described as baseline models) for hardware implementation. In order to address these issues, we propose an estimation model that includes the estimation of these channels and characterizes the performance of the HS in terms of the sensing-throughput tradeoff.

\section{Proposed APPROACH}

\section{A. Frame structure}

In order to incorporate the estimation of the involved channels, a novel frame structure is proposed in Fig. 2, according to which, $\tau_{\mathrm{est}, h_{\mathrm{p}, 1}}$ and $\tau_{\mathrm{est}, h_{\mathrm{p}, 2}}$ are utilized for estimating $h_{\mathrm{p}, 1}$ and $h_{\mathrm{p}, 2}$ by the ST and $\mathrm{SR}^{1}$, respectively. Besides that, $\tau_{\mathrm{est}} h_{\mathrm{p}, 3}$ is used for estimating the $h_{\mathrm{p}, 3}$. Since the ST considers the estimation based on the pilot symbols transmitted by the ST that already exist in the secondary system, no time resources are allocated for the estimation of $h_{\mathrm{s}}$. However, it is possible that the time interval between two control-based transmissions

\footnotetext{
${ }^{1}$ In order to accomplish this, it is assumed that both the ST and the SR align themselves to the control-based transmission from the PT.
} 
is large as compared to $T$. Under such conditions, the frame structure followed by the secondary system can be adapted from the one proposed in Fig. 2 in such a way that channel estimation is restricted to particular frames and the remaining frames follow the conventional structure, i.e., sensing followed by data transmission. Hence, our proposed approach presents a general framework and is adaptable to different controlbased configurations followed by various primary systems. For scenarios, where the PT and the PR represent a single entity, i.e., interchangeably act as transmitter and receiver, the first two slots $\tau_{\mathrm{est},} h_{\mathrm{p}, 1}$ and $\tau_{\mathrm{est}, h_{\mathrm{p}, 3}}$ of the ST can be combined into one, cf. Fig.2.

\section{B. Channel Estimation}

Here, we present the estimation of the interacting channels. In this work, we follow similar approach for the channel estimation to the one described in [9], [13], according to which, it is logical to employ a received power-based estimation for the sensing and the interference channels, and a pilot-based estimation for the access channel.

Sensing Channel $\left(h_{p, 1}\right)$ : The ST estimates the sensing channel by estimating the received power from the PT during $\tau_{\mathrm{est}, h_{\mathrm{p}, 1}}$. With $\tau_{\mathrm{est}, h_{\mathrm{p}, 1}} f_{\mathrm{s}}$ samples used for estimation, the estimated received power $\hat{P}_{\mathrm{Rx}, \mathrm{ST}, h_{\mathrm{p}, 1}}=\sum_{n=1}^{\tau_{\mathrm{est},} h_{\mathrm{p}, 1} f_{\mathrm{s}}} \mid h_{\mathrm{p}, 1} x_{\mathrm{PT}}[n]+$ $\left.w[n]\right|^{2}$ follows a $\mathcal{X}^{2}$ distribution. The cumulative distribution function (CDF) of $\hat{P}_{\mathrm{Rx}, \mathrm{ST}, h_{\mathrm{p}, 1}}$ is characterized as

$$
F_{\hat{P}_{\mathrm{R} x, \mathrm{ST}, h_{\mathrm{p}, 1}}}(x)=1-\Gamma\left(\frac{\tau_{\mathrm{est}, h_{\mathrm{p}, 1}} f_{\mathrm{s}}}{2}, \frac{\tau_{\mathrm{est}, h_{\mathrm{p}, 1}} f_{\mathrm{s}} x}{2 P_{\mathrm{Rx}, \mathrm{ST}, h_{\mathrm{p}, 1}}}\right) .
$$

In order to improve the detector's performance, the samples $f_{\mathrm{s}} \tau_{\mathrm{est}}, h_{\mathrm{p}, 1}$ considered for the estimation can also be utilized for the sensing $f_{\mathrm{s}} \tau_{\mathrm{sen}}$, cf. Fig. 2 . In this sense, hereafter, the characterization of the detector incurs the time interval $\tau_{\text {est, } h_{\mathrm{p}, 1}}$.

Access Channel $\left(h_{s}\right)$ : The pilot signal received from the SR undergoes matched filtering and demodulation at the ST, hence, we employ a pilot-based estimation at the ST to acquire the knowledge of the access channel. The maximum-likelihood estimate of $\hat{h}_{\mathrm{s}}$ presented by [14] is unbiased, efficient, i.e., achieves a Cramér-Rao bound with equality and asymptotic variance $\mathbb{E}\left[\left|h_{\mathrm{s}}-\hat{h}_{\mathrm{s}}\right|^{2}\right]=\frac{\sigma_{\mathrm{v}}^{2}}{2 N_{\mathrm{s}}}$, where $N_{\mathrm{s}}$ denotes the number of pilot symbols. As a result, $\hat{h}_{\mathrm{s}}$ conditioned on $h_{\mathrm{s}}$ follows a Gaussian distribution $\hat{h}_{\mathrm{s}} \mid h_{\mathrm{s}} \sim \mathcal{N}\left(h_{\mathrm{s}}, \frac{\sigma_{\mathrm{w}}^{2}}{2 N_{\mathrm{s}}}\right)$. Consequently, the estimated power gain $\left|\hat{h}_{\mathrm{s}}\right|^{2}$ follows a non-central chi-squared $\mathcal{X}_{1}^{2}\left(\lambda_{\mathrm{s}}, 1\right)$ distribution with 1 degree of freedom and noncentrality parameter $\lambda_{\mathrm{s}}=\frac{2 N_{\mathrm{s}}\left|h_{\mathrm{s}}\right|^{2}}{\sigma_{\mathrm{w}}^{2}}$. For analytical tractability, we consider the following approximation.

Approximation 1: For all degrees of freedom, the $\mathcal{X}_{1}^{2}$ distribution can be approximated by a Gamma distribution [15]. The parameters of the Gamma distribution are obtained by matching the first two central moments to those of $\mathcal{X}_{1}^{2}$.

Following Approximation 1, the CDF of $\left|\hat{h}_{\mathrm{s}}\right|^{2}$ is characterized as

$$
F_{\left|\hat{h}_{\mathrm{s}}\right|^{2}}(x) \approx 1-\Gamma\left(a, \frac{x}{b}\right),
$$

$$
\text { where } a=\frac{\left(1+\lambda_{\mathrm{s}}\right)^{2}}{2+4 \lambda_{\mathrm{s}}} \text { and } b=\frac{\sigma_{\mathrm{s}}^{2}\left(2+4 \lambda_{\mathrm{s}}\right)}{\left(1+\lambda_{\mathrm{s}}\right)} .
$$

Interference Channel $\left(h_{p, 2}\right)$ : Besides the access channel, the knowledge of the interference channel to the SR from the PT is required for determining the SU throughput. It is worthy to note that the expression $\left|h_{\mathrm{p}, 2}\right|^{2} \sigma_{\mathrm{s}}^{2}+\sigma_{\mathrm{w}}^{2}$ in $R_{1}$ and $R_{3}$, cf. (9) and (11), which corresponds to the interference and the noise power, represents $P_{\mathrm{Rx}, \mathrm{SR}, h_{\mathrm{p}, 2}}$. Hence, by estimating $\hat{P}_{\mathrm{Rx}, \mathrm{SR}, h_{\mathrm{p}, 2}}$, we are able to jointly characterize the interference and the noise, and consequently characterize $R_{1}$ and $R_{3}$. In this view, the SR estimates received power by listening to the controlbased transmission from the PT, cf. Fig. 2. Similar to the sensing channel, the CDF of the estimated interference power received $\hat{P}_{\mathrm{Rx}, \mathrm{SR}, h_{\mathrm{p}, 2}}$ at the $\mathrm{ST}$ is characterized as

$$
F_{\hat{P}_{\mathrm{R}, \mathrm{SR}, h_{\mathrm{p}, 2}}}(x)=1-\Gamma\left(\frac{\tau_{\mathrm{est}, h_{\mathrm{p}, 2}} f_{\mathrm{s}}}{2}, \frac{\tau_{\mathrm{est}, h_{\mathrm{p}, 2}} f_{\mathrm{s}} x}{2 P_{\mathrm{Rx}, \mathrm{SR}, h_{\mathrm{p}, 2}}}\right) .
$$

Interference Channel $\left(h_{p, 3}\right)$ : Lastly, the estimation of the interference channel between the ST and the PR is essential for employing the power control at the ST. Like the sensing channel, the ST estimates $\left|h_{\mathrm{p}, 3}\right|^{2}$ by listening to the controltransmission from the PR. The received power estimated $\left(P_{\left.\mathrm{Rx}, \mathrm{ST}, h_{\mathrm{p}, 3}\right)}\right)$ from $\tau_{\mathrm{est}, h_{\mathrm{p}, 3}} f_{\mathrm{s}}$ samples follows $\mathcal{X}^{2}$ distribution

$$
F_{\hat{P}_{\mathrm{Rx}, S T, h_{\mathrm{p}, 3}}}(x)=1-\Gamma\left(\frac{\tau_{\mathrm{est}, h_{\mathrm{p}, 3}} f_{\mathrm{s}}}{2}, \frac{\tau_{\mathrm{est}, h_{\mathrm{p}, 3}} f_{\mathrm{s}} x}{2 P_{\mathrm{Rx}, \mathrm{ST}, h_{\mathrm{p}, 3}}}\right) .
$$

\section{Characterization of Performance Parameters}

It is clear that the estimation of the involved channels translates to the variations in the performance parameters, which include detection probability $\mathrm{P}_{\mathrm{d}}$ at the $\mathrm{ST}$, interference power received $P_{\mathrm{Rx}, \mathrm{PR}}$ at the $\mathrm{PR}$ and $\mathrm{SU}$ throughput $R_{\mathrm{s}}$ at the SR. In particular, the variations in $P_{d}$ and $P_{\text {Rx,PR }}$ may cause severe interference to the primary system, hence, seriously degrading the performance of $\mathrm{CR}$ systems. This renders the existing constraints defined by the ideal model inaccurate. Motivated by this fact, we capture these variations by proposing new outage constraints $\rho_{\mathrm{d}}$ and $\rho_{\text {cont }}$ on the $\mathrm{P}_{\mathrm{d}}$ and $\hat{P}_{\mathrm{Rx}, \mathrm{PR}}$, respectively, as PU constraints for the HS. These constraints are defined as

$$
\begin{aligned}
& \mathbb{P}\left(\mathrm{P}_{\mathrm{d}}\left(\hat{P}_{\mathrm{Rx}, \mathrm{ST}, h_{\mathrm{p}, 1}}\right) \leq \overline{\mathrm{P}}_{\mathrm{d}}\right) \leq \rho_{\mathrm{d}},
\end{aligned}
$$

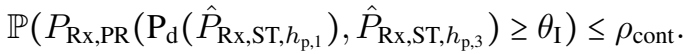

In contrast to the ideal model, in the current context, it is reasonable to consider an outage over the two constraints, cf. (6) and (7), jointly in terms of the aggregate interference $\hat{P}_{\mathrm{Rx}, \mathrm{PR}}$ (which includes the noise power) at the PR. In this regard, (20) is written as

$$
\begin{aligned}
& \mathbb{P}[\mathbb{P}\left(\mathcal{H}_{1}\right) \cdot \overbrace{\left(\frac{\hat{P}_{\mathrm{Rx}, \mathrm{ST}, h_{\mathrm{p}, 3}}-\sigma_{\mathrm{w}}^{2}}{\sigma_{\mathrm{s}}^{2}}\right)}^{\left|h_{\mathrm{p}, 3}\right|^{2}} \\
& \left.\times\left(\left(1-\mathrm{P}_{\mathrm{d}}\right) P_{\mathrm{T} \mathrm{x}, \mathrm{ST}, \text { full }}+\mathrm{P}_{\mathrm{d}} P_{\mathrm{Tx}, \mathrm{ST}, \mathrm{cont}}\right) \geq \theta_{\mathrm{I}}\right] \leq \rho_{\text {cont }} .
\end{aligned}
$$


It is worth noticing the fact that $\mathbb{P}(\cdot)$ in (21) jointly characterizes the variations due to $\mathrm{P}_{\mathrm{d}}$ and $P_{\mathrm{Rx}, \mathrm{PR}}$.

We proceed further by characterizing the CDF of $\mathrm{P}_{\mathrm{d}}, P_{\mathrm{Rx}, \mathrm{PR}}$ and $R_{\mathrm{s}}$. This is done by transforming the CDFs of the estimated parameters, characterized previously in (14), (15), (17) and (18). To begin with, we consider the CDF of the $P_{d}$ in order to respect the constraint on the detection probability defined in (19).

Lemma 1: The CDF of $\mathrm{P}_{\mathrm{d}}$ is characterized as [9]

$$
F_{\mathrm{P}_{\mathrm{d}}}(x)=1-\Gamma\left(\frac{\tau_{\mathrm{est}, h_{\mathrm{p}, 1}} f_{\mathrm{s}}}{2}, \frac{\tau_{\mathrm{est}, h_{\mathrm{p}, 1}} f_{\mathrm{s}} \tau_{\mathrm{sen}} f_{\mathrm{s}} \mu}{4 P_{\mathrm{Rx}, \mathrm{ST}, h_{\mathrm{p}, 1}} \Gamma^{-1}\left(\frac{\tau_{\mathrm{sen}}}{2}, x\right)}\right),
$$

where $\Gamma^{-1}(\cdot, \cdot)$ is inverse function of regularized upper incomplete Gamma function [12].

Next, we characterize the CDF of the $P_{\mathrm{Rx}, \mathrm{PR}}$ in order to satisfy the outage constraint defined in (20).

Lemma 2: The CDF of $\hat{P}_{\mathrm{Rx}, \mathrm{PR}}$ is characterized as (23, see the top of next page).

Besides that, since the variations in $\mathrm{P}_{\mathrm{d}}, \hat{P}_{\mathrm{Rx}, \mathrm{ST}, h_{\mathrm{p}, 1}},\left|\hat{h}_{\mathrm{s}}\right|^{2}$ and $\hat{P}_{\mathrm{Rx}, \mathrm{ST}, h_{\mathrm{p}, 3}}$ translate to the variations in $R_{\mathrm{s}}$, these variations are captured in terms of the expected throughput. More specifically, the variations in $\hat{P}_{\mathrm{Rx}, \mathrm{ST}, h_{\mathrm{p} .1}},\left|\hat{h}_{\mathrm{s}}\right|^{2}$ and $\hat{P}_{\mathrm{Rx}, \mathrm{ST}, h_{\mathrm{p} .3}}$ result in variations in capacities $\mathrm{C}_{0}, \mathrm{C}_{1}, \mathrm{C}_{2}$ and $\mathrm{C}_{3}$, cf. (8), (9), (10) and (11). In this view, we characterize the probability density functions (pdfs) for $\mathrm{C}_{0}, \mathrm{C}_{1}, \mathrm{C}_{2}$ and $\mathrm{C}_{3}$ in the following Lemmas.

Lemma 3: The pdf of $\mathrm{C}_{0}$ is defined as [9]

$$
\begin{gathered}
f_{\mathrm{C}_{0}}(x)=2^{x} \ln 2 \frac{\left(2^{x}-1\right)^{a_{1}-1}}{\Gamma\left(a_{1}\right) b^{a_{1}}} \exp \left(-\frac{2^{x}-1}{b_{1}}\right), \\
\text { where } a_{0}=a \text { and } b_{0}=\frac{P_{\mathrm{Tx}, \mathrm{ST}, \text { full }}}{\sigma_{\mathrm{w}}^{2}} b,
\end{gathered}
$$

where $a$ and $b$ are defined in (16).

Lemma 4: The pdf of $\mathrm{C}_{1}$ is defined as [9]

$$
\begin{gathered}
f_{\mathrm{C}_{1}}(x)=2^{x} \ln 2 \frac{\left(2^{x}-1\right)^{a_{0}-1} \Gamma\left(a_{0}+a_{1}\right)}{\Gamma\left(a_{0}\right) \Gamma\left(a_{1}\right) b_{0}^{a_{0}} b_{1}^{a_{1}}}\left(\frac{1}{b_{1}}+\frac{2^{x}-1}{b_{0}}\right), \\
\text { where } a_{1}=\frac{N_{\mathrm{p}, 2}}{2} \text { and } b_{1}=\frac{2 P_{\mathrm{Rx}, \mathrm{SR}, h_{\mathrm{p}, 2}}}{\sigma_{\mathrm{w}}^{2} N_{\mathrm{p}, 2}},
\end{gathered}
$$

and $a_{0}, b_{0}$ are defined in (25).

Following the characterization of the pdfs for $\mathrm{C}_{0}$ and $\mathrm{C}_{1}$, the pdfs for $\mathrm{C}_{2}$ and $\mathrm{C}_{3}$ can be obtained by substituting $P_{\mathrm{Tx}, \mathrm{ST}, \mathrm{cont}}$ for $P_{\mathrm{Tx}, \mathrm{ST}, \text { full }}$ in (24) and (26).

Lemma 5: The pdf of $\mathrm{C}_{2}$ is defined as

$$
f_{\mathrm{C}_{2}}(x)=2^{x} \ln 2 \frac{\left(2^{x}-1\right)^{a_{2}-1}}{\Gamma\left(a_{2}\right) b^{a_{2}}} \exp \left(-\frac{2^{x}-1}{b_{2}}\right),
$$

where $a_{2}=\frac{\left(1+\lambda_{\mathrm{s}}\right)^{2}}{2+4 \lambda_{\mathrm{s}}}$ and $b_{2}=\frac{P_{\mathrm{Tx}, \mathrm{ST}, \mathrm{cont}}}{\sigma_{\mathrm{w}}^{2}} \frac{\sigma_{\mathrm{s}}^{2}\left(2+4 \lambda_{\mathrm{s}}\right)}{\left(1+\lambda_{\mathrm{s}}\right)}$.

Lemma 6: The pdf of $\mathrm{C}_{3}$ is defined as

$$
f_{\mathrm{C}_{3}}(x)=2^{x} \ln 2 \frac{\left(2^{x}-1\right)^{a_{2}-1} \Gamma\left(a_{2}+a_{1}\right)}{\Gamma\left(a_{2}\right) \Gamma\left(a_{1}\right) b_{2}^{a_{2}} b_{1}^{a_{1}}}\left(\frac{1}{b_{1}}+\frac{2^{x}-1}{b_{2}}\right) \text {, }
$$

where $a_{1}, b_{1}$ and $a_{2}, b_{2}$ are defined in (27) and (29), respectively.
Subsequently, the variations arising due to $\mathrm{P}_{\mathrm{d}}$ are captured by considering $F_{\mathrm{P}_{\mathrm{d}}}$ characterized in Lemma 1 . As a result, the expected throughput is given by (31), where $\mathbb{E}_{\Omega}[\cdot]$ denotes the expectation over $\Omega$, where $\Omega \in\left\{\mathrm{P}_{\mathrm{d}}, \mathrm{C}_{0}, \mathrm{C}_{1}, \mathrm{C}_{2}, \mathrm{C}_{3}\right\}$, (31, see the top of next page).

\section{Sensing-Throughput Tradeoff}

Here, we establish a sensing-throughput tradeoff for the proposed approach that incorporates variations in the performance parameters.

Theorem 1: The expected achievable SU throughput subject to an outage constraint on detection probability at the ST and an outage constraint on interference power at the PR given by

$$
\begin{aligned}
R_{\mathrm{s}}\left(\tilde{\tau}_{\text {sen }}\right) & =\max _{\tau_{\text {sen }}, P_{\mathrm{T}, \mathrm{ST} \text {, cont }}} \mathbb{E}_{\Omega}\left[R_{\mathrm{s}}\left(\tau_{\text {sen }}\right)\right] \\
\text { s.t. } & (19),(20) .
\end{aligned}
$$

Proof: In order to solve the constrained optimization problem, we make the following realistic assumption. The primary system attains sufficient protection when high detection probability is achieved by the ST. In this sense, it is reasonable to consider first the constraint on the detection probability with desired $\overline{\mathrm{P}}_{\mathrm{d}}$ and $\rho_{\mathrm{d}}$, cf. (19). Hence, we use this assumption to obtain an expression of $\mu$ [9]

$$
\mu \geq \frac{4 P_{\mathrm{Rx}, \mathrm{ST}, h_{\mathrm{p}, 1}} \Gamma^{-1}\left(1-\rho_{\mathrm{d}}, \frac{\tau_{\mathrm{est}, h_{\mathrm{p}, 1}} f_{\mathrm{s}}}{2}\right) \Gamma^{-1}\left(\overline{\mathrm{P}}_{\mathrm{d}}, \frac{\tau_{\mathrm{sen}} f_{\mathrm{s}}}{2}\right)}{\tau_{\mathrm{est}, h_{\mathrm{p}, 1}} \tau_{\mathrm{sen}}\left(f_{\mathrm{s}}\right)^{2}} .
$$

Next, using the constraint (20), the controlled transmit power at the SR is determined as

$$
F_{P_{\mathrm{Rx}, \mathrm{PR}}}\left(\theta_{\mathrm{I}}\right) \geq \rho_{\text {cont }}
$$

Solving numerically (34) yields $P_{\mathrm{Tx}, \mathrm{ST}, \mathrm{cont}}$ for the HS. Finally, by substituting $\mu$ and $P_{\mathrm{TX}, \mathrm{ST}, \mathrm{cont}}$ computed in (34) and (33), and using the density functions of $\mathrm{P}_{\mathrm{d}}, \mathrm{C}_{0}, \mathrm{C}_{1}, \mathrm{C}_{2}$ and $\mathrm{C}_{3}$ determined in Lemma 1, Lemma 3, Lemma 4, Lemma 5 and Lemma 6 yield an expression of $\mathbb{E}_{\Omega}\left[R_{\mathrm{s}}\right]$ as a function of $\tau_{\mathrm{sen}}$, cf. (31). Solving numerically $\mathbb{E}_{\Omega}\left[R_{\mathrm{s}}\right]$ against the $\tau_{\text {sen }}$ delivers $\tilde{\tau}_{\text {sen }}$ that achieves the maximum expected SU throughput.

Remark 1: Herein, using the estimation model, we establish a fundamental relation between estimation time (regulates the variations in the detection probability and interference power received at the PR according to the PU constraint), sensing time (represents the detector's performance) and SU throughput, this relationship is characterized as an estimationsensing-throughput tradeoff.

\section{NumericAl ANALYSIS}

In this section, we evaluate the performance of the HS for the proposed approach. In this view, we perform simulations: (i) to validate the expressions obtained in the previous section, (ii) to analyze the performance loss incurred due to channel estimation. In order to illustrate the performance loss, we consider the ideal model to benchmark the performance of the proposed approach. Unless stated explicitly, the choice of parameters given in Table $\mathrm{I}$ is considered for analysis. 


$$
\begin{gathered}
F_{P_{\mathrm{R}, \mathrm{PR}}}(x)=\int_{0}^{1} \Gamma\left(\frac{\tau_{\mathrm{est}, h_{\mathrm{p}, 3}} f_{\mathrm{s}}}{2},\left(\frac{x \sigma_{\mathrm{s}}^{2}}{h_{\mathrm{p}, 1} \cdot\left(\left(1-\mathrm{P}_{\mathrm{d}}\right) P_{\mathrm{Tx}, \mathrm{ST}, \mathrm{full}}+\mathrm{P}_{\mathrm{d}} P_{\mathrm{Tx}, \mathrm{ST}, \mathrm{cont}}\right)}+\sigma_{\mathrm{w}}^{2}\right) \cdot \frac{\tau_{\mathrm{est}, h_{\mathrm{p}, 3}} f_{\mathrm{s}}}{2 P_{\mathrm{Rx}, \mathrm{ST}, h_{\mathrm{p}, 3}}}\right) d F_{\mathrm{P}_{\mathrm{d}}} \cdot \\
\mathbb{E}_{\Omega}\left[R_{\mathrm{s}}\left(\tau_{\mathrm{sen}}\right)\right]=\frac{T-\tau_{\mathrm{est}, h_{\mathrm{p}, 3}-\tau_{\mathrm{sen}}}^{T} \cdot\left[\left(1-\mathrm{P}_{\mathrm{fa}}\right) \cdot \mathbb{P}\left(\mathcal{H}_{0}\right) \cdot \mathbb{E}_{\mathrm{C}_{0}}\left[\mathrm{C}_{0}\right]+\left(1-\mathbb{E}_{\mathrm{P}_{\mathrm{d}}}\left[\mathrm{P}_{\mathrm{d}}\right]\right) \cdot \mathbb{P}\left(\mathcal{H}_{1}\right) \cdot \mathbb{E}_{\mathrm{C}_{1}}\left[\mathrm{C}_{1}\right]+\mathrm{P}_{\mathrm{fa}} \cdot \mathbb{P}\left(\mathcal{H}_{0}\right) \cdot \mathbb{E}_{\mathrm{C}_{2}}\left[\mathrm{C}_{2}\right]+\right.}{\left.\mathbb{E}_{\mathrm{P}_{\mathrm{d}}}\left[\mathrm{P}_{\mathrm{d}}\right] \cdot \mathbb{P}\left(\mathcal{H}_{1}\right) \cdot \mathbb{E}_{\mathrm{C}_{3}}\left[\mathrm{C}_{3}\right]\right] .}
\end{gathered}
$$

\begin{tabular}{|c|c|c|}
\hline \multicolumn{3}{|c|}{ PARAMETERS FOR NUMERICAL ANALYSIS } \\
\hline Parameter & Definition & Value \\
\hline$f_{\mathrm{s}}$ & Sampling Frequency & $1 \mathrm{MHz}$ \\
\hline$T$ & Frame Duration & $100 \mathrm{~ms}$ \\
\hline$\tau_{\text {est, } h_{\mathrm{p} 1}}$ & Estimation time for the channel $h_{\mathrm{p}, 1}$ & $1 \mathrm{~ms}$ \\
\hline$\tau_{\mathrm{est}, ~} h_{\mathrm{p}, 2}$ & Estimation time for the channel $h_{\mathrm{p}, 2}$ & $1 \mathrm{~ms}$ \\
\hline$\tau_{\text {est, }, h_{\mathrm{p}, 3}, 2}$ & Estimation time for the channel $h_{\mathrm{p}, 3}$ & $1 \mathrm{~ms}$ \\
\hline$\left|h_{\mathrm{p}, 1}\right|^{2}$ & Power gain for channel $h_{\mathrm{p}, 1}$ & $-120 \mathrm{~dB}$ \\
\hline$\left|h_{\mathrm{p}, 2}\right|^{2}$ & Power gain for channel $h_{\mathrm{p}, 2}$ & $-120 \mathrm{~dB}$ \\
\hline$\left|h_{\mathrm{p}, 3}\right|^{2}$ & Power gain for channel $h_{\mathrm{p}, 3}$ & $-100 \mathrm{~dB}$ \\
\hline$\left|h_{\mathrm{s}}\right|^{2}$ & Power gain for channel $h_{\mathrm{s}}$ & $-80 \mathrm{~dB}$ \\
\hline$\theta_{\mathrm{I}}$ & Interference threshold & $-110 \mathrm{dBm}$ \\
\hline$\rho_{\text {cont }}$ & Outage constraint on interference power at PR & 0.1 \\
\hline$\rho_{\mathrm{d}}$ & Outage constraint on detection probability & 0.1 \\
\hline$\sigma_{\mathrm{s}}^{2}$ & Transmit power at PT and PR & $10 \mathrm{dBm}$ \\
\hline$\sigma_{\mathrm{w}}^{2}$ & Noise power at ST, SR and PR & $-100 \mathrm{dBm}$ \\
\hline$\overline{\mathrm{P}}_{\mathrm{d}}$ & Detection probability threshold & 0.9 \\
\hline $\mathbb{P}\left(\mathcal{H}_{0}\right)$ & Occurrence Probability for hypothesis $\mathcal{H}_{0}$ & 0.2 \\
\hline$P_{\mathrm{Tx}, \mathrm{ST} \text {.full }}$ & Transmit power at ST & $0 \mathrm{dBm}$ \\
\hline$N_{\mathrm{s}}$ & Number of pilot symbols & 10 \\
\hline
\end{tabular}

TABLE I

We first analyze the performance of the HS in terms of a sensing-throughput tradeoff, cf. Theorem 1, corresponding to the Ideal Model (IM) and Estimation Model (EM) by fixing $\tau_{\text {est, } h_{\mathrm{p}, 1}}=\tau_{\mathrm{est}, h_{\mathrm{p}, 2}}=\tau_{\mathrm{est}, h_{\mathrm{p}, 3}}=1 \mathrm{~ms}$, cf. Fig. 3 . With the inclusion of channel estimation in the frame structure, the ST procures no throughput at the SR for the interval $\tau_{\mathrm{est}, h_{\mathrm{p}, 1}}$. As indicated by the margin between the IM and the EM, a certain performance degradation is witnessed by the EM due to the incorporation of channel estimation. Moreover, the sensingthroughput tradeoff yields a suitable sensing time $\tilde{\tau}_{\text {sen }}$ that achieves the maximum performance in terms of SU throughput $R_{\mathrm{s}}\left(\tilde{\tau}_{\text {sen }}\right)$. Hereafter, we consider theoretical expressions for the analysis and intend to operate at suitable sensing time.

Following the previous discussions, it is well-known that the combination of interweave and underlay systems is intended to enhance the performance of the HS, hence, it is worthy to acquire insights on the performance gain in terms of the achievable SU throughput. In this sense, we study the variations of achievable SU throughput corresponding to the channel between the ST and the PR, cf. Fig. 1. Before proceeding with the analysis, it is essential to understand that the performance of the underlay system decreases with the increase in the channel gain $\left|h_{\mathrm{p}, 3}\right|^{2}$ [13]. In this view, we categorize the channel gain $\left|h_{\mathrm{p}, 3}\right|^{2} \in[-110,-90] \mathrm{dBm}$ in three different regimes: (i) Regime I, (ii) Regime II and (iii) Regime III, cf. Fig. 4. Under Regime I, large channel gain $\left|h_{\mathrm{p}, 3}\right|^{2}>-93 \mathrm{~dB}$ causes control power to fall below a certain

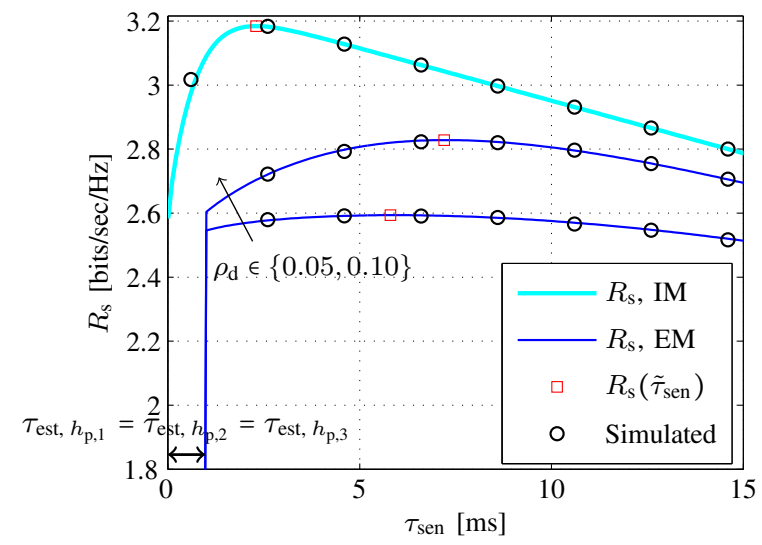

Fig. 3. Sensing-throughput tradeoff for the Ideal Model and Estimation Model (EM) for $\tau_{\mathrm{est}, h_{\mathrm{p}, 1}}=\tau_{\mathrm{est}, h_{\mathrm{p}, 2}}=\tau_{\mathrm{est}, h_{\mathrm{p}, 3}}=1 \mathrm{~ms}$.

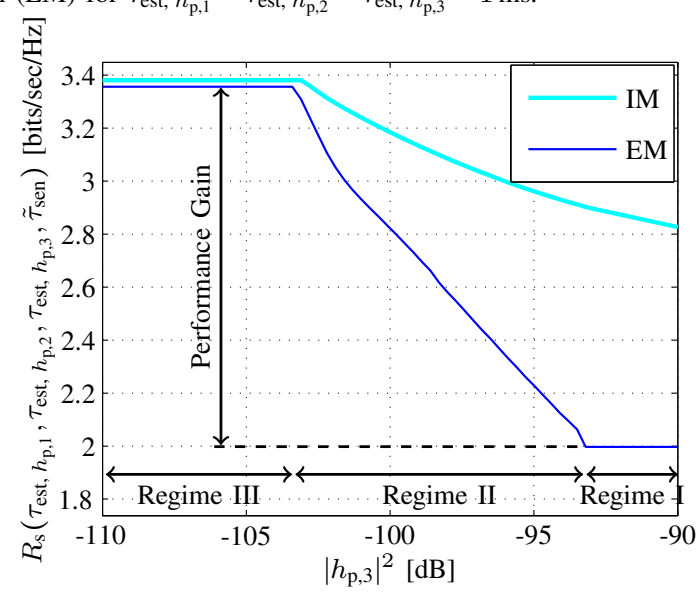

Fig. 4. Achievable SU throughput for the IM and EM versus path loss $\left|h_{\mathrm{p}, 3}\right|^{2}$ where the system is operating at suitable sensing time $\tilde{\tau}_{\text {sen }}$ and the estimation time is fixed to $\tau_{\mathrm{est}, h_{\mathrm{p}, 1}}=\tau_{\mathrm{est}, h_{\mathrm{p}, 2}}=\tau_{\mathrm{est}, h_{\mathrm{p}, 3}}=1 \mathrm{~ms}$ and $\rho_{\mathrm{d}}=0.10$.

level $P_{\mathrm{Tx}, \mathrm{ST}, \text { cont }} \leq-20 \mathrm{dBm}$, for the considered value of the channel gain $\left|h_{\mathrm{s}}\right|^{2}=-80 \mathrm{~dB}$ over the access channel, such a low power transmission do not translate to an effective performance gain to the HS. As a result, no benefits are attained from the underlay system while operating in this regime, hence, the HS operates as an interweave system. In contrast to that, the Regime II $\left(-103 \mathrm{~dB}<\left|h_{\mathrm{p}, 3}\right|^{2}<-93 \mathrm{~dB}\right)$ witnesses a significant performance gain as HS procures benefits from both underlay and interweave systems. Moreover, it is observed that, no performance gain is attained below a certain channel gain $\left|h_{\mathrm{p}, 3}\right|^{2}<-103 \mathrm{~dB}$ (Regime III). This is due to the fact that the ST is limited by the maximum transmit power, i.e., beyond $-103 \mathrm{~dB}, P_{\mathrm{Tx}, \mathrm{ST}, \mathrm{cont}}=P_{\mathrm{Tx}, \mathrm{ST}, \text { full }}$. 


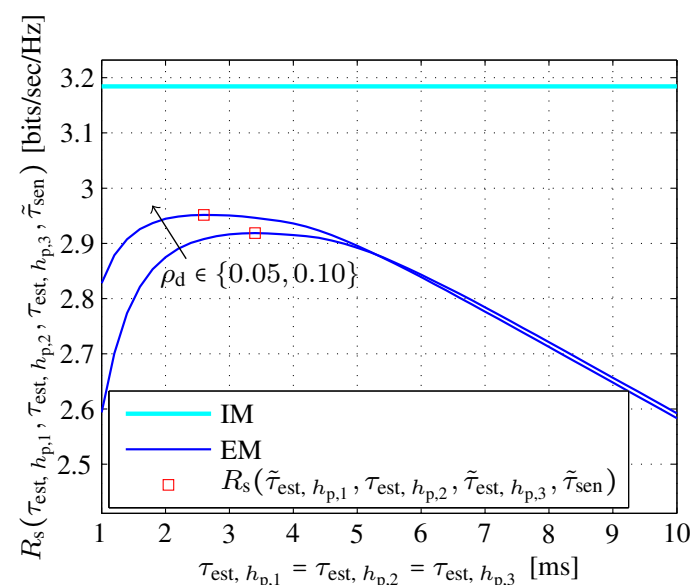

Fig. 5. Achievable SU throughput versus the estimation time $\tau_{\mathrm{est}, h_{\mathrm{p}, 1}}=$ $\tau_{\mathrm{est}, h_{\mathrm{p}, 2}}=\tau_{\mathrm{est}, h_{\mathrm{p}, 3}}$ operating at the suitable sensing time $\tilde{\tau}_{\mathrm{sen}}$.

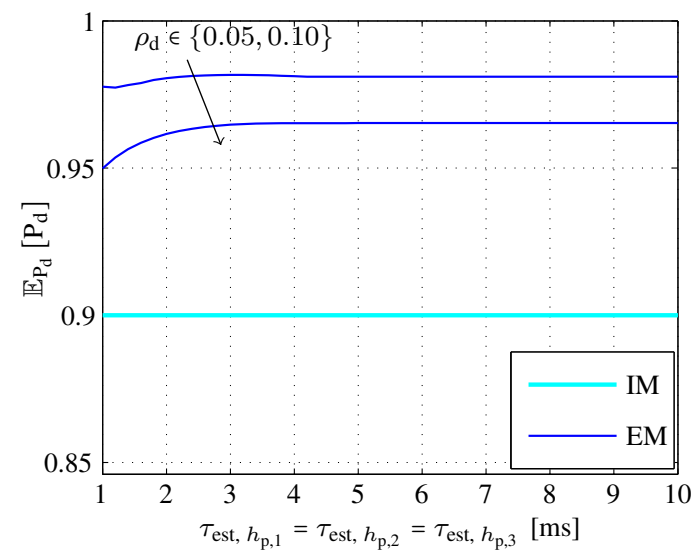

Fig. 6. Detection probability versus $\tau_{\mathrm{est}, h_{\mathrm{p}, 1}}=\tau_{\mathrm{est}, h_{\mathrm{p}, 2}}=\tau_{\mathrm{est}, h_{\mathrm{p}, 3}}$ operating at the suitable sensing time $\tilde{\tau}_{\text {sen }}$.

From this discussion, it can be concluded that the interference tolerance capability of the underlay system and the detection capability of the interweave system incorporated by the HS can be transformed into significant performance gain only in situations where channel to the PR is below a certain level, for instance $\left|h_{\mathrm{p}, 3}\right|^{2}<-93 \mathrm{dBm}$ for the considered case.

Besides maximizing the SU throughput over the sensing time, it is interesting to observe the variation of the achievable throughput with the estimation time. To simplify the analysis, we consider that $\tau_{\mathrm{est}, h_{\mathrm{p}, 1}}=\tau_{\mathrm{est}, h_{\mathrm{p}, 2}}=\tau_{\mathrm{est}, h_{\mathrm{p}, 3}}$. Corresponding to the estimation model, Fig. 5 reveals the estimation-sensingthroughput tradeoff, cf. Remark 1. This effect can be explained as follows, the variations due to the estimation of $\left|h_{\mathrm{p}, 1}\right|^{2}$ and $\left|h_{\mathrm{p}, 3}\right|^{2}$ causes variations in $\mathrm{P}_{\mathrm{d}}$ and $\hat{P}_{\mathrm{Rx}, \mathrm{PR}}$, these variations are captured using the outage constraints, hence, a small increase in $\tau_{\text {est, } h_{\mathrm{p}, 1}}\left(=\tau_{\mathrm{est}, h_{\mathrm{p}, 2}}=\tau_{\mathrm{est}, h_{\mathrm{p}, 3}}\right)$ leads to a significant performance improvement in terms of SU throughput, however increasing the estimation time beyond $\tilde{\tau}_{\mathrm{est}, h_{\mathrm{p}, 1}}\left(=\tilde{\tau}_{\mathrm{est}, h_{\mathrm{p}, 2}}=\right.$ $\left.\tilde{\tau}_{\mathrm{est},} h_{\mathrm{p}, 3}\right)$ slightly contributes to the performance improvement and largely consumes the time resources.

To procure further insights, we analyze the detector's performance with the estimation time. For the EM, it is observed that, for all values of the estimation time, $\mathbb{E}_{\mathrm{P}_{d}}\left[\mathrm{P}_{\mathrm{d}}\right]$ strictly stays above $\overline{\mathrm{P}}_{\mathrm{d}}$, cf. Fig. 6 . This indicates that the proposed approach yields a reasonable performance of the detector incorporated in the HS.

\section{CONCLUSION}

In this paper, we have investigated the performance of cognitive radio as a hybrid system that utilizes the benefits of both underlay and interweave paradigms from a deployment perspective. It has been argued that the lack of knowledge of the involved channels renders the existing models unsuitable for the performance characterization. In this view, an analytical framework that incorporates channel estimation and subsequently captures the effect of imperfect channel knowledge has been established. More importantly, by means of an estimation-sensing-throughput tradeoff, suitable estimation time and suitable sensing time that achieves the maximum SU throughput have been determined. In our future work, we plan to extend the proposed analysis to include the effect of channel fading on the performance of the hybrid systems.

\section{REFERENCES}

[1] A. Goldsmith, S. Jafar, I. Maric, and S. Srinivasa, "Breaking Spectrum Gridlock With Cognitive Radios: An Information Theoretic Perspective," Proceedings of the IEEE, vol. 97, no. 5, pp. 894-914, May 2009.

[2] S. Sharma, T. Bogale, S. Chatzinotas, B. Ottersten, L. Le, and X. Wang, "Cognitive Radio Techniques under Practical Imperfections: A Survey," IEEE Communications Surveys Tutorials, vol. 17, no. 4, pp. 1858-1884, Fourthquarter 2015.

[3] Y.-C. Liang, Y. Zeng, E. Peh, and A. T. Hoang, "Sensing-Throughput Tradeoff for Cognitive Radio Networks," IEEE Transactions on Wireless Communications, vol. 7, no. 4, pp. 1326-1337, April 2008.

[4] X. Kang, Y.-C. Liang, H. Garg, and L. Zhang, "Sensing-based spectrum sharing in cognitive radio networks," IEEE Transactions on Vehicular Technology, vol. 58, no. 8, pp. 4649-4654, Oct 2009.

[5] J. Oh and W. Choi, "A hybrid cognitive radio system: A combination of underlay and overlay approaches," in IEEE 72nd Vehicular Technology Conference Fall (VTC 2010-Fall), Sept 2010, pp. 1-5.

[6] S. Senthuran, A. Anpalagan, and O. Das, "Throughput analysis of opportunistic access strategies in hybrid underlay-overlay cognitive radio networks," IEEE Transactions on Wireless Communications, vol. 11, no. 6, pp. 2024-2035, June 2012.

[7] X. Jiang, K.-K. Wong, Y. Zhang, and D. Edwards, "On Hybrid OverlayUnderlay Dynamic Spectrum Access: Double-Threshold Energy Detection and Markov Model," IEEE Transactions on Vehicular Technology, vol. 62, no. 8, pp. 4078-4083, Oct 2013.

[8] S. Sharma, S. Chatzinotas, and B. Ottersten, "A hybrid cognitive transceiver architecture: Sensing-throughput tradeoff," in CROWNCOM, Jun. 2014.

[9] A. Kaushik, S. K. Sharma, S. Chatzinotas, B. Ottersten, and F. K. Jondral, "Sensing-Throughput Tradeoff for Interweave Cognitive Radio System: A Deployment-Centric Viewpoint," IEEE Transactions on Wireless Communications, vol. PP, no. 99, 2016.

[10] S. Kay, Fundamentals of Statistical Signal Processing: Detection theory, ser. Prentice Hall Signal Processing Series. Prentice-Hall PTR, 1998.

[11] R. Tandra and A. Sahai, "SNR Walls for Signal Detection," IEEE Journal of Selected Topics in Signal Processing, vol. 2, no. 1, pp. 4-17, Feb 2008.

[12] I. S. Gradshteyn and I. M. Ryzhik, Table of Integrals, Series, and Products, 6th ed. San Diego, CA: Academic Press., 2000.

[13] A. Kaushik, S. K. Sharma, S. Chatzinotas, B. Ottersten, and F. K. Jondral, "On the Performance Analysis of Underlay Cognitive Radio Systems: A Deployment Perspective," IEEE Transactions on Cognitive Communications and Networking, (submitted).

[14] W. Gifford, M. Win, and M. Chiani, "Antenna subset diversity with non-ideal channel estimation," IEEE Transactions on Wireless Communications, vol. 7, no. 5, pp. 1527-1539, May 2008.

[15] M. Abramowitz and I. A. Stegun, Handbook of Mathematical Functions with Formulas, Graphs, and Mathematical Tables, ninth Dover printing, tenth GPO printing ed. New York: Dover, 1964. 